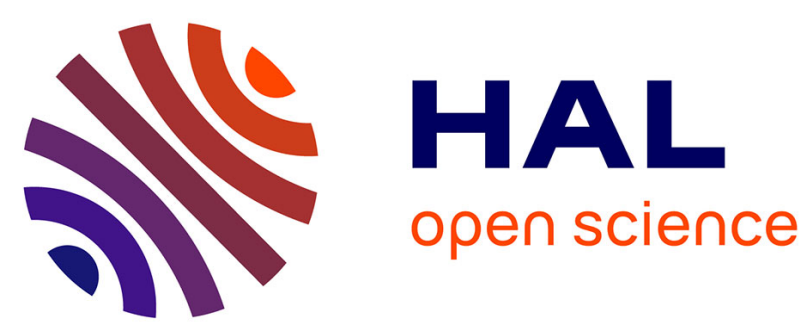

\title{
Le contrat unique
}

Jacques Barthélémy, Gilbert Cette

\section{- To cite this version:}

Jacques Barthélémy, Gilbert Cette. Le contrat unique: Une auberge espagnole. Revue de l'OFCE, 2016, 146, pp.43 - 65. 10.3917/reof.146.0043 . hal-03389350

\section{HAL Id: hal-03389350 \\ https: / hal-sciencespo.archives-ouvertes.fr/hal-03389350}

Submitted on 20 Oct 2021

HAL is a multi-disciplinary open access archive for the deposit and dissemination of scientific research documents, whether they are published or not. The documents may come from teaching and research institutions in France or abroad, or from public or private research centers.
L'archive ouverte pluridisciplinaire HAL, est destinée au dépôt et à la diffusion de documents scientifiques de niveau recherche, publiés ou non, émanant des établissements d'enseignement et de recherche français ou étrangers, des laboratoires publics ou privés. 


\title{
LE CONTRAT UNIQUE UNE AUBERGE ESPAGNOLE ${ }^{1}$
}

\author{
Jacques Barthélémy \\ Avocat conseil en droit social, fondateur du cabinet Barthélémy Avocats \\ Gilbert Cette \\ Professeur d'économie associé à l'Université d'Aix-Marseille
}

Le débat sur le contrat unique découle le plus souvent du constat d'une dualité du marché du travail, avec d'un côté des salariés qui seraient très protégés, les CDI et tout particulièrement les fonctionnaires, et de l'autre côté les actifs alternant emplois précaires peu protégés et périodes de chômage. Ce contraste traduit des inégalités flagrantes, avec des conséquences sociales et économiques importantes.

En réponse à cette dualité, des propositions sont souvent avancées qui consisteraient à créer un "contrat unique » atténuant les écarts de statut et de droits entre emplois précaires (CDD et intérim) et emplois en CDI. Mais ce concept de " contrat unique » est souvent peu défini. C'est même une auberge espagnole si l'on prend en considération les différences importantes de contenu constatées dans les propos des uns ou des autres!

Les trois objectifs déclarés de la proposition de contrat unique sont : (i) de réduire les inégalités de statuts liées à la coexistence de contrats dits précaires (CDD et intérim) et de CDI ; (ii) de réduire la complexité et les incertitudes coûteuses de la judiciarisation des licenciements ; (iii) d'internaliser en partie le coût social des licenciements. Notre analyse montre qu'un contrat unique ne peut pas répondre véritablement à ces objectifs, mieux servis par d'autres voies, et qu'il présenterait des risques juridiques majeurs.

Mots clés : réformes structurelles, marché du travail, droit du travail, négociations collectives, syndicats.

1. Nous reprenons ici des analyses plus longuement développées avec P.-Y. Verkindt dans : Barthélémy, Cette et Verkindt (2006) ; Barthélémy, Cette et Verkindt (2007a) ; Barthélémy, Cette et Verkindt (2007b). Ces analyses ont été reprises dans Barthélémy et Cette (2015). 


\section{Introduction}

Le débat sur le contrat unique est récurrent en France comme dans d'autres pays européens. À l'origine, le constat d'une dualité du marché du travail, avec d'un côté des salariés qui seraient très protégés, les CDI et tout particulièrement les fonctionnaires, et de l'autre côté les actifs alternant emplois précaires peu protégés et périodes de chômage. Ce contraste traduit des inégalités flagrantes, avec des conséquences sociales et économiques importantes, concernant par exemple l'accès à la formation professionnelle ou à des prêts ou au logement locatif.

En réponse à cette dualité, des propositions sont souvent avancées qui consisteraient à créer un "contrat unique " atténuant les écarts de statuts et de droits entre emplois précaires (CDD et intérim) et emploi en CDI. Mais ce concept de "contrat unique » est souvent peu défini. C'est même une auberge espagnole si l'on prend en considération les différences importantes de contenu constatées dans les propos des uns ou des autres! Certains rejettent sur les juristes et les décideurs politiques la responsabilité de lui trouver un contenu opérationnel et considèrent que leur incapacité à y parvenir traduit un manque de détermination lié à une opposition sourde des syndicats ou une insensibilité coupable à l'égard d'inégalités pourtant criantes. Cette position nous paraît tout aussi inacceptable que celle qui consisterait à rejeter sur les laboratoires médicaux ou le corps médical la responsabilité des ravages provoqués par certaines maladies sous prétexte qu'ils ne s'efforceraient pas réellement de trouver LE médicament adapté à chacune !

Il convient de souligner que si de nombreux économistes et politiques se sont déclarés favorables au contrat unique, à l'opposé, de nombreux juristes de droit social manifestent pour le moins une grande réserve concernant cette proposition. Il serait tout aussi déplacé de faire aux économistes le procès de ne pas reconnaître la nécessité du droit social que de faire aux juristes de droit social celui d'ignorer la nécessité de l'efficacité économique. Ceci incite donc à traiter de la question du contrat unique dans une approche multidisciplinaire.

Quand on parle d'un concept que l'on veut spécifique comme le contrat unique, il faut être précis. Aussi, notre analyse va-t-elle s'appuyer sur des propositions qui ont pour premier mérite d'avoir 
répondu à cette exigence de précision, contrairement à d'autres qui demeurent péremptoires et sans contenu. De ce fait, l'analyse qui suit s'appuie sur trois rapports d'économistes qui ont préconisé l'instauration d'un contrat de travail unique se substituant à l'actuelle coexistence des CDD et CDI : Blanchard et Tirole (2003), Camdessus (2004) et Cahuc et Kramarz (2005), les propositions de ce dernier rapport étant plus détaillées que celles avancées dans les deux précédents. Ces trois rapports sont en France au cœur de la quasi-totalité des développements émanant des promoteurs du contrat unique. Nous les avons retenus également en raison à la fois de la qualité des arguments avancés et de la notoriété de leurs auteurs.

Les trois objectifs principaux de la proposition de contrat unique dans ces rapports sont les suivants (cf. Encadré 1) :

- Réduire les inégalités de statuts actuellement renforcées par la coexistence de contrats dits précaires (CDD et intérim) et de CDI. Cette inégalité de statuts peut être indirectement source d'autres inégalités, comme par exemple celle concernant l'accès à des prêts ou au logement (2.) ;

- Réduire la complexité et les incertitudes coûteuses de la judiciarisation des licenciements (3.) ;

- Internaliser en partie le coût social (prestations chômage et destruction de capital humain) des licenciements, ce coût social allant de pair avec un faible effort de formation des titulaires d'emplois précaires. Cela revient à responsabiliser sur ce point les entreprises. Une telle responsabilisation peut avoir pour effet favorable de davantage sécuriser les parcours professionnels. Ceci étant, les trois rapports évoqués envisagent cette responsabilisation par la seule voie financière (4.).

Or, un contrat unique ne peut pas répondre véritablement à ces objectifs, mieux servis par d'autres voies (évoquées dans la section conclusive 5.). 


\section{Encadré 1. Le contrat unique proposé dans trois rapports}

Blanchard et Tirole (2003), p. 11 (voir aussi p. 47) :

« D'où notre troisième recommandation : il est essentiel de retourner à un système unique, mais modulé de façon à faciliter l'entrée des jeunes sur le marché du travail et la réinsertion des chômeurs, sans créer les effets de seuil présents dans le système actuel. Ceci peut être fait en utilisant deux instruments. Le premier, qui existe déjà, est celui d'une période d'essai, pendant laquelle l'entreprise et le salarié peuvent terminer la relation de travail, sans droits ni devoirs. Le second est l'introduction d'une augmentation progressive des droits des licenciés et des devoirs des entreprises en fonction à la fois de l'ancienneté dans l'entreprise et de l'expérience récente du salarié sur le marché du travail ».

Camdessus (2004), p. 92 :

" Le groupe considère qu'une piste intéressante sur laquelle les partenaires sociaux devraient réfléchir est la création d'un contrat unique. Convenablement conçu, il permettrait de résoudre de fait la dualisation du marché du travail.

La forme précise de ce contrat est naturellement à débattre. Mais une idée peut être avancée : la suppression du CDD et la création d'un contrat unique à durée indéterminée dans lequel les droits relatifs à la protection d'emploi et à l'indemnisation se renforceraient progressivement. Sans tenter ici de préciser les contours d'un tel contrat, on voit bien que les anciens cas de recours au CDD constitueraient des cas de motif réel et sérieux de rupture dans les premiers temps du contrat. Ainsi s'intègreraient sans heurts les embauches antérieurement opérées en CDD et cette nouvelle souplesse faciliterait l'insertion durable dans l'entreprise. Les procédures de licenciement connaîtraient une progressivité comparable, permettant à l'entreprise de gérer son personnel en fonction de ses besoins réels et non par des pratiques contestables de ruptures successives. En cas de rupture précoce, l'actuelle prime de précarité pourrait être majorée ».

\section{Cahuc et Kramarz (2005), pp. 145-146 :}

"Le contrat unique est à durée indéterminée. Il n'y a donc plus d'embauche en contrat à durée déterminée, y compris dans les secteurs où existe la possibilité de 'contrats déterminés d'usage' ».

"Le contrat de travail unique que nous proposons a pour objet de sécuriser et d'homogénéiser les parcours professionnels en incitant les entreprises à tenir compte de la valeur sociale des emplois afin d'assurer un véritable droit au reclassement aux salariés. Dans cette perspective, le contrat de travail unique possède trois caractéristiques principales : (1) C'est un contrat à durée indéterminée ; (2) En cas de rupture du contrat, l'employeur paye une indemnité, versée au salarié, et une contribution de solidarité, versée, à l'instar de la «contribution Delalande », aux pouvoirs publics ; (3) La signature du contrat de travail offre l'assurance d'un accompagnement personnalisé et d'un revenu de remplacement en cas de perte d'emploi ». 


\section{Réduire les inégalités de statuts}

Signalons tout d'abord que ces propositions de contrat unique n'évoquent pas le statut des agents du secteur public dont bénéficie en France plus d'un salarié sur quatre. Or, la principale inégalité, de droit et de fait, est moins celle existant entre CDI et des CDD du secteur privé que celle existant entre d'un côté le statut de la fonction publique et de nombreuses entreprises publiques, avec en particulier la sécurité quasi totale d'emploi, de l'autre la situation des travailleurs du secteur privé, qu'ils soient en CDI ou en CDD. Il nous semble pour le moins paradoxal d'avancer des propositions de réduction des écarts de statut entre CDI et CDD sans évoquer cet aspect, qui conduirait cependant à remettre totalement en cause le statut du personnel de la fonction publique, la difficulté politique de cet aspect ayant sans doute rendu timorés les auteurs des trois rapports évoqués ci-dessus comme les autres intervenants sur la question.

$\mathrm{Au}$ demeurant, l'idée de contrat unique repose sur un contresens majeur : le CDI ne sécurise pas plus l'emploi que le CDD et parfois même c'est l'inverse. En effet, un contrat à durée indéterminée - et ceci ne vaut pas que pour celui du travail - peut toujours être rompu à tout moment par l'une ou l'autre des parties car tout engagement à vie est prohibé. Cela implique seulement l'obligation de respecter un préavis. Les exigences entourant la rupture de ce contrat particulier qu'est celui du travail sont liées à une protection de la partie faible (qui conditionne ce que l'on a coutume d'appeler « ordre public économique »), particulier ici dès lors que l'objet de ce contrat c'est l'Homme dont doivent être respectés les droits fondamentaux et les libertés individuelles (Conv. EDH et Déclaration des droits de l'Homme et du citoyen de 1789). Pour autant, le licenciement est toujours possible sauf exception (exemple : les représentants du personnel en raison de leur mandat dans l'intérêt de la collectivité de travail) même si cela se traduit potentiellement par des indemnités pouvant être élevées. Au contraire, la rupture anticipée d'un contrat à durée déterminée est juridiquement contre-nature et elle n'est, de ce fait, possible, s'agissant du contrat de travail, que dans des cas très limités (faute grave et cas de force majeure). La raison de la préférence pour le CDI se trouve donc ailleurs, essentiellement dans le sentiment de la précarité. $\mathrm{Si}$, sociologiquement, les conditions sont réunies pour que le 
CDD ne soit pas perçu comme un sous-contrat, ses bénéficiaires potentiels le privilégient ( $C f$. ci-dessous l'exemple du domaine sportif professionnel).

Par ailleurs, l'idée de contrat unique ne prend pas en compte des besoins spécifiques auxquels le CDD apporte une réponse : (i) ceux de l'employeur qui peut avoir besoin de salariés pour des durées spécifiques et spécifiées : activités saisonnières, remplacement de salariés absents pour des périodes connues (congés de maladie, de maternité...) ; (ii) ceux du salarié qui peut vouloir travailler sur une période donnée, avec une réelle protection sur cette période. À cet égard, le CDD d'usage que l'on supprimerait de ce fait correspond dans certaines activités, telles le sport professionnel, à une demande pressante des travailleurs eux-mêmes. Dans le cadre du rapport Karaquillo (2015) sollicité par le Ministre des Sports et visant à optimiser le statut des sportifs de haut niveau et des sportifs professionnels, tous les syndicats de sportifs ont demandé que l'on introduise une présomption irréfragable de CDD, estimant le CDI moins protecteur !

Le CDD correspond à la fois à un réel besoin des entreprises et dans certains cas à une attente des salariés. Pour autant, la précarité qu'il est susceptible de générer amène à ne pas encourager son recours, afin que ce dernier se rapproche le plus possible des situations où il répond à un réel besoin. Tel est au demeurant le sens de la Directive européenne de 1999 qui met en œuvre un accord collectif CES-UNICE. Cette directive, relative au CDD, se distingue par le fait que la validité du recours au CDD est admise par le droit communautaire si le droit interne respecte les exigences d'un motif légitime lié à la nature de l'emploi, d'une durée maximum et d'un nombre limité de renouvellements. Si, pour les partenaires sociaux européens dont l'accord est annexé à la directive, le CDI doit être privilégié, le CDD a néanmoins une légitimité.

Le CDD sécurise davantage l'employeur contre le risque de judiciarisation à la fin du contrat. Cela ne doit pas pour autant justifier son recours pour des situations auxquelles il n'est pas censé répondre, car cela entraînerait un affaiblissement du rôle protecteur du droit social. Aussi, le principe actuel des durées maxima des CDD devrait être conservé, ce qui n'empêche pas une réflexion sur le niveau souhaitable de telles durées, notamment leur adaptation à différents types d'emplois mais aussi sur le nombre de renouvelle- 
ments. Ceci d'autant que la directive précitée laisse des espaces d'assouplissement dont pourrait se saisir le droit légal interne, à moins qu'on ouvre la voie à de possibles dérogations par accord collectif (de branche seulement?) dans les limites de ce qu'autorise le droit communautaire.

Par ailleurs, le recours au CDD doit être découragé par un surcoût spécifique, bénéficiant à la fois au salarié et aux organismes de gestion des indemnisations du chômage :

- Concernant les salariés, la prime de précarité est une contrepartie souhaitable à la réduction de la contestation possible en fin de son contrat à la seule hypothèse de requalification. Ici encore, le bon niveau de cette indemnité de précarité peut faire l'objet de discussion, spécialement de négociations collectives ;

- Concernant les organismes de gestion des indemnisations du chômage, une taxe spécifique portant sur les emplois précaires (intérim et CDD) est souhaitable compte tenu de la disproportion actuelle entre le coût de l'indemnisation chômage des personnes ayant acquis leur droit dans des emplois précaires et les cotisations chômage prélevées sur l'emploi précaire. Cet aspect est développé plus loin.

Il serait alors logique que, dans les professions et les emplois où il est d'usage de recourir au CDD et où - par voie de conséquence celui-ci est considéré comme un mode normal de contractation, cette taxe pourrait être écartée.

\section{Réduire la complexité et les incertitudes coûteuses de la judiciarisation des licenciements}

Le risque de judiciarisation s'accroîtrait avec le contrat unique contrairement à ce qu'estiment leurs zélateurs. Hors période d'essai, qui s'oppose à l'obligation de motiver le licenciement ? Une telle motivation est d'ailleurs conforme aux engagements internationaux de la France, notamment ${ }^{2}$ à la Convention $n^{\circ} 158$ de l'OIT ratifiée par la France en 1989 (Cf. Encadré 2). Et si le licenciement est motivé, la contestation du motif est alors possible, et le risque de judiciarisation ne peut être écarté. Une discontinuité inévitable

2. Mais pas seulement, $c f$. Barthelemy, Cette et Verkindt $(2006,2007)$. 
apparaît ici : au-delà d'une certaine ancienneté, la séparation doit être motivée pour un CDI, ce qui n'est par nature pas le cas pour un emploi précaire. Cette discontinuité ne peut disparaître ni être réduite. En outre, de l'article 30 (titre IV relatif à la solidarité) de la charte des droits fondamentaux de l'Union européenne (version consolidée du 26 avril 2012), il ressort que tout travailleur a droit à une protection contre le licenciement injustifié.

\section{Encadré 2.}

Extraits de la Convention 158 de l'Organisation internationale du travail, et les mauvais exemples du CNE et du CPE

L'article 4 de cette Convention précise : «Un travailleur ne devra pas être licencié sans qu'il existe un motif valable de licenciement lié à l'aptitude ou à la conduite du travailleur ou fondé sur les nécessités du fonctionnement de l'entreprise, de l'établissement ou du service ». L'article 7 ajoute : "Un travailleur ne devra pas être licencié pour des motifs liés à sa conduite ou à son travail avant qu'on ne lui ait offert la possibilité de se défendre contre les allégations formulées, à moins que l'on ne puisse pas raisonnablement attendre de l'employeur qu'il lui offre cette possibilité ». L'article 8 ajoute : «Un travailleur qui estime avoir fait l'objet d'une mesure de licenciement injustifiée aura le droit de recourir contre cette mesure devant un organisme impartial tel qu'un tribunal, un tribunal du travail, une commission d'arbitrage ou un arbitre ». L'article 9-1-a précise même : « la charge de prouver l'existence d'un motif valable de licenciement tel que défini à l'article 4 de la présente convention devra incomber à l'employeur ».

Cette nécessité est illustrée par l'aventure du Contrat nouvelle embauche (CNE). Le CNE permettait aux entreprises de 20 salariés et moins le licenciement sans motivation les deux premières années suivant l'embauche. Instauré en 2005, il a été considéré par l'OIT en 2007 non conforme à la convention 158 de cette même organisation, convention ratifiée par la France en $1989^{3}$. Il a pour cette raison été abrogé en février 2008.

L'autre aventure du Contrat première embauche (CPE), simultanée à celle du CNE, traduit les risques d'une non prise en compte des attentes des personnes concernées. Le CPE transposait le principe du CNE pour l'ensemble des entreprises du secteur privé, mais pour la seule population de moins de 26 ans. Instauré en janvier 2006, ce dispositif fut retiré définitivement en avril de la même année à la suite d'une importante

3. Voir sur ce point le Rapport du Directeur Général, OIT (2007). 
mobilisation de jeunes. Signalons d'ailleurs que, pour les mêmes raisons que le CNE, le CPE se serait probablement révélé en contradiction avec le droit international.

Ces tentatives de réformes vouées à l'échec ont des effets désastreux. Elles décrédibilisent en effet, tant auprès des employeurs que des salariés et des organisations syndicales, le processus de réforme lui-même. Elles font par ailleurs perdre un temps précieux dans la mise en œuvre de ce processus.

Blanchard et Tirole (2003) font la proposition de ne pas laisser au juge la possibilité d'apprécier le réel caractère économique des licenciements faits sous ce motif ( $C f$. Encadré 3). Cette proposition est dangereuse car le motif économique pourrait être abusivement invoqué pour réduire le risque de contestation. Elle peut même se heurter au droit imprescriptible de tout citoyen de recourir au juge. Remarquons de plus que le nombre de ruptures de contrats liées à une cause économique est très inférieur à celui des ruptures pour cause personnelle.

\section{Encadré 3. La place du juge dans l'analyse des procédures de licenciement des trois rapports}

Blanchard et Tirole (2003) :

p. 10. : «Le rôle du juge est alors de vérifier, si le salarié en fait la demande, qu'un licenciement pour raison économique n'a pas été transformé en licenciement pour faute ou pour démission. De même, il importe de vérifier que le licenciement ne soit pas motivé sur une discrimination basée sur la race, le sexe ou l'appartenance syndicale, que les délais de préavis aient été respectés, etc. Par contre, les juges ne doivent pas se substituer au jugement de l'entreprise dans sa gestion, car ils n'en ont ni la compétence ni en général l'information nécessaire, sans mentionner le manque de critères précis guidant leur intervention. Si l'entreprise reconnaît l'acte de licenciement et est prête à en supporter les coûts, le rôle du juge doit alors être confiné à la vérification de la procédure ».

p. 36: «Nous avons insisté sur le rôle des juges parce que nous pensons qu'ils ont un rôle nécessaire à jouer. Mais il est important de noter que, dans notre argumentation, ce rôle ne s'étend pas aux licenciements économiques. Dans ce cas, si l'entreprise est prête à payer les taxes et indemnités de licenciements, nous ne voyons pas le rôle que le processus judiciaire a à jouer ». 
p. 47 : «Le licenciement économique doit, lui, donner droit aux allocations chômage pour le ou les salariés licenciés, et au paiement de taxes et indemnités de licenciement par l'entreprise. Ces paiements responsabilisent l'entreprise face aux licenciements. Si, dans ces conditions, l'entreprise est prête à effectuer ces paiements, nous ne saisissons pas la logique sous-jacente à l'examen et à l'invalidation de cette décision par l'appareil judiciaire. On peut penser que le système en place à l'heure actuelle, qui permet aux juges de contester la décision de licenciement de l'entreprise, trouve sa justification dans l'absence de responsabilisation financière des entreprises. La solution nous paraît être de responsabiliser les entreprises, non de demander aux juges de contester la décision de l'entreprise ».

\section{Camdessus (2004) :}

pp. 99 et 100 : «Il serait judicieux de bien cantonner juridiquement les deux types de dispositifs: d'une part la rupture de contrat, où la rupture abusive se résout en dommages et intérêts; d'autre part les procédures collectives. Concrètement, il s'agirait de supprimer la spécificité relative au licenciement pour motif économique. Ainsi, un défaut, ou un déficit, dans le processus de consultation se traduira par une sanction pénale adaptée à la gravité de la faute commise par l'employeur, et s'agissant du contrat, seule donnerait lieu à sanction l'absence de motif réel et sérieux ».

Cahuc et Kramarz (2005) :

p. 155, sous l'intitulé de sous-partie «Garantie de reclassement et abandon de la notion de licenciement économique»: "Dans un contexte où l'employeur prend en compte la valeur sociale de l'emploi dans sa décision, paye pour que le salarié soit pris en charge efficacement par l'État aidé par des professionnels, le paiement de la contribution de solidarité et le respect de la procédure de licenciement devraient constituer des critères suffisants pour juger si un licenciement repose sur une cause réelle et sérieuse. Ainsi, la logique introduite par le nouveau système simplifie naturellement la réglementation des licenciements dans la mesure où il n'est plus nécessaire de réserver un traitement particulier au licenciement économique ».

"L'abandon de la notion de licenciement économique » ou tout du moins de son particularisme préconisé par Cahuc et Kramarz (2005) est-il possible juridiquement et surtout souhaitable? Le licenciement économique est le seul motif de licenciement qui ne met pas en cause la personne. C'est la raison pour laquelle il est inapproprié de le supprimer, notamment d'un point de vue «moral». Mais conserver la notion de licenciement économique n'interdit pas d'en simplifier les procédures, en particulier dans les 
cas de licenciements collectifs, pour en réduire les coûts et incertitudes. Il n'est toutefois pas inutile de rappeler que la créativité en ce domaine est réduite du fait du droit communautaire. Cependant, l'ANI du 11 janvier 2013 a fait des propositions bienvenues de sécurisation juridique de PSE en cas de licenciements collectifs, ces propositions ayant été transcrites dans le Code du travail par la loi du 14 juin 2013. Et puis, à nouveau, il ne faut pas perdre de vue les contraintes pouvant émaner du droit communautaire. Or, existent des dispositions relatives au traitement des difficultés des entreprises qui affectent des systèmes particuliers de garanties en cas de licenciement économique. La notion de cause réelle et sérieuse a potentiellement un champ plus vaste que celle de licenciement abusif.

La simplification des procédures juridiques de licenciement, sans doute souhaitable, diffère de la problématique du contrat unique. Cette simplification ne doit pas réduire les droits des salariés. Celle que nous avançons dans Barthelemy et Cette (2015) répond à cette attente. Elle responsabilise de façon équilibrée les acteurs locaux en proposant que la gestion des conflits puisse échapper, grâce au recours à l'arbitrage, aux juridictions de droit commun, à condition d'être organisée par accord collectif majoritaire et que soient, en amont, optimisées les conditions de la conciliation.

Pour autant, le droit actuel semble encourager une telle judiciarisation, du fait de l'asymétrie des risques. En première analyse, le salarié risque peu à engager une procédure tandis que l'entreprise risque d'être condamnée à indemniser le préjudice du licenciement sur le fondement de l'absence de motif réel et sérieux. Cette asymétrie n'est pas nécessairement inéquitable si l'on considère l'état de subordination du salarié. On ne saurait oublier que le licenciement est une décision unilatérale de l'employeur qui, comme toute décision économique, constitue une prise de risque. Par ailleurs, la subordination juridique inhérente à la relation de travail justifie amplement l'abandon de toute tentative de parallélisme juridique entre la situation dépendante du salarié et les pouvoirs de l'employeur.

Enfin, on ne saurait oublier que, lorsque le salarié agit, il est déjà hors de l'entreprise. 
Même s'il est impensable d'écarter toute exigence pour licencier, d'autant plus en raison de la loyauté nécessaire dans les relations individuelles de travail, une autre formulation que la référence au motif "sérieux" pourrait prospérer dans la perspective d'une cohérence avec la responsabilité de l'employeur au plan économique, liée à la liberté d'entreprendre mais dans le respect des exigences précitées du droit communautaire.

Les délais de prescription de l'action en justice méritent un examen critique. La limitation du risque pour l'employeur devrait s'accompagner de l'obligation faite au salarié d'agir en justice dans des délais raisonnables. Le mouvement législatif va d'ailleurs dans le sens de leur réduction, la loi du 14 juin 2013 transcrivant dans le Code du travail les propositions de l'ANI du 11 janvier 2013 ayant réduit à deux années au lieu de 5 précédemment la plupart des délais de prescription. De tels délais de deux années paraissent encore longs, comparés à ceux qui existent dans d'autres pays. Cela étant, l'ordre public de protection exige un délai de prescription relativement long.

Une voie parfois évoquée pour une plus grande sécurisation juridique des licenciements est l'instauration de plafonds d'indemnisation. Cet aspect mérite une réflexion spécifique et, à cet égard, les dispositions introduites dans la Loi Macron à l'occasion de son passage en seconde lecture à l'Assemblée nationale en juin 2015 paraissaient juridiquement risquées, essentiellement s'agissant d'une évaluation différente du préjudice suivant la taille de l'entreprise. Le Conseil constitutionnel a d'ailleurs censuré ce dernier aspect.

La justice est un droit fondamental de l'Homme et c'est un principe de l'Union européenne. Le droit à la réparation, par le juge, du préjudice subi du fait de l'autre est inaliénable. Cela vaut bien évidemment pour le salarié victime d'un comportement de son employeur. Ce droit est dans le droit du travail exacerbé à la fois parce que le pouvoir de l'employeur dans l'entreprise est normatif, justifié par l'intérêt de l'entreprise, et parce que le contrat a comme objet l'Homme, dont la dignité doit être respectée. C'est du reste la raison pour laquelle l'idée qu'en sortant de la convention 158 de l'OIT on peut écarter toute obligation pour l'employeur de motiver le licenciement, est absurde. 
Cela étant, l'incertitude dans laquelle se trouve l'employeur d'évaluer a priori le montant des sommes qu'il devra au salarié dont il envisage de rompre le contrat est indiscutablement cause de frilosité dans le dynamisme entrepreneurial, ce qui nuit à l'efficacité économique donc à l'emploi. Le nier relève d'une approche purement idéologique. Au demeurant, la longueur des procès, induite pour partie de cette incertitude et plus généralement de l'insécurité juridique née de l'inadéquation du droit social au contexte dans lequel il prospère ainsi que de la boulimie législative, se traduit par une atteinte aux droits fondamentaux, spécialement ceux issus de la Conv. EDH.

Voilà pourquoi il peut paraître pertinent de fixer - forfaitairement ou par un maximum - les dommages et intérêts liés à l'absence de cause sérieuse de licenciement (encadré 4). On pouvait raisonnablement s'interroger sur la constitutionnalité d'une telle construction législative en raison du droit à réparation intégrale du préjudice subi. La position du Conseil constitutionnel qui ne condamne le texte que sur le terrain des différences entre entreprises suivant leur taille permet donc de conclure à la constitutionnalité de barèmes, donc des montants liés à l'ancienneté. Pour autant, à l'interrogation sur l'exigence de réparation intégrale du préjudice, il eût été intéressant d'opposer la nécessaire distinction entre l'absence de cause sérieuse, création du législateur et à ce titre pouvant peut-être faire l'objet d'un barème et le licenciement abusif par lequel la réparation intégrale s'impose. Le droit de la faute inexcusable en matière d'accident du travail ou de maladie professionnelle dans lequel la réparation forfaitaire existe parce qu'on est sur le terrain de l'assurance peut servir à cet égard de témoignage dès lors que la réparation intégrale du préjudice y subsiste néanmoins dans certaines circonstances ${ }^{4}$.

Une telle distinction permettait du reste d'écarter la critique de stratégies de l'employeur fondées sur la mesure de la réalité du risque au regard des bénéfices de l'infraction, car le comportement abusif entraînerait, quoi qu'il en soit, la réparation intégrale du préjudice. Au demeurant, l'attitude de l'employeur exclusivement

4. Dans l'ANI du 11 janvier 2008, transposé dans le Code du travail en juin de la même année, les partenaires sociaux ont proposé de créer la rupture conventionnelle à partir d'un commun accord des parties, qui a pour effet de réduire le risque judiciaire et l'incertitude financière. 
conditionnée par ce calcul atteste d'un abus de droit, au vu de sa définition en doctrine concrétisée par la fictivité de l'objet de la décision. En gros, si un employeur utilise un droit en étant respectueux de la lettre des textes mais que la finalité poursuivie est étrangère à son objet, il y a abus de droit. La distinction entre absence de cause sérieuse et abus de droit pourrait s'inscrire dans cette logique. L'abus de droit étant un concept parfaitement cerné en droit, cette distinction pourrait être utilement opérée.

\section{Encadré 4. Les plafonds d'indemnisation : une voie juridiquement risquée}

L'idée selon laquelle il serait possible d'instaurer un plafond d'indemnisation pourrait paraître séduisante. Elle serait en première analyse un facteur de sécurité pour le salarié (qui connaîtrait ainsi par avance les sommes auxquelles il pourrait prétendre) et pour l'employeur (qui pourrait prévoir le coût potentiel d'une rupture et pourquoi pas provisionner les sommes correspondantes en comptabilité). Les dispositions ajoutées à la Loi Macron à l'occasion de son passage en seconde lecture à l'Assemblée nationale en juin 2015 introduisent une barémisation des indemnités prononcées pour les licenciements dénués de cause réelle et sérieuse. Il convient d'analyser ces dispositions. Mais auparavant, deux questions se posent : la première est de savoir si ce dispositif n'existe pas déjà et la seconde est relative à la source d'une telle disposition.

\section{La prévisibilité de l'indemnisation n'est pas inconnue du droit} du travail

Il faut en effet distinguer selon que le licenciement est ou non fondé sur une cause réelle et sérieuse. Dans le premier cas, que la cause soit personnelle ou économique, la prévisibilité existe déjà puisque l'employeur sait qu'en licenciant un salarié pour un motif réel et sérieux, il sera tenu au versement d'une indemnité de licenciement dont le quantum est prévu par l'article L 1234-9 du Code du travail, d'une indemnité de congés payés (qu'il calculera aisément sur la base du salaire du salarié et du nombre de mois travaillés entre le $1^{\text {er }}$ juin précédent et le licenciement) et d'une indemnité compensatrice de préavis (dont la durée est calculée et donc parfaitement prévisible compte tenu de l'ancienneté du salarié). Ces indemnités légales peuvent être majorées par application des textes conventionnels applicables. Ce sont alors les montants ou les durées conventionnellement prévues qui seront applicables, mais l'employeur les connaît ou est supposé les connaître par la simple consultation des conventions et accords collectifs applicables dans l'entreprise. La seule incertitude résulterait de l'invocation par l'employeur d'une faute grave ou d'une faute lourde, qui lui permet- 
trait d'en refuser le paiement. En pareil cas, l'action du salarié en justice tendant à faire juger l'absence de faute grave ou lourde pourrait conduire à une décision condamnant l'employeur à payer les indemnités susvisées. Si indétermination du coût du licenciement il y avait dans une telle hypothèse, elle résulterait du choix de l'employeur de s'appuyer sur une faute qualifiée, à ses risques et périls en quelque sorte.

La visibilité de ce qui l'attend lorsqu'il prend le risque de rompre un contrat ou de dénoncer un accord est une exigence majeure pour le chef d'entreprise, donc pour satisfaire à l'intérêt général. On ne peut sans doute pas limiter le montant des sommes destinées à réparer un préjudice (à cet égard la comparaison avec le régime des accidents du travail/ maladies professionnelles (AT/MP) n'est pas convaincante car on est là sur le terrain de l'assurance). Le dispositif du projet de Loi Macron, consistant à fournir aux juges un barème indicatif, est toutefois de nature à favoriser le règlement des litiges dès la conciliation.

2. Le droit d'obtenir l'indemnisation du préjudice subi du fait du comportement fautif de l'employeur n'est pas négociable

Tout autre est la situation où le licenciement, acte unilatéral de l'employeur faut-il le rappeler, serait jugé non fondé. En ce cas, c'est le juge qui appréciera le montant de l'indemnisation. La question porte alors sur le fait de savoir si, d'une façon ou d'une autre, un plafond d'indemnisation peut être instauré soit par la voie conventionnelle soit par l'effet d'une modification législative.

Sur le premier point, il faut distinguer la source contractuelle et la source conventionnelle collective. Dans l'état actuel du droit du travail, la réponse est assurément négative s'agissant du contrat car le salarié ne saurait renoncer par avance à se prévaloir du droit du licenciement en application du Code du travail ${ }^{5}$. Une clause de ce type serait nulle, d'une nullité d'ordre public et réputée non écrite et la Cour de cassation l'a rappelé fermement dans trois décisions du 30 mars $2005^{6}$ (un salarié ne peut valablement renoncer pendant la durée du contrat par avance, au droit de se prévaloir des règles légales du licenciement). Ce qu'un contrat ne peut faire, une convention collective ou un accord collectif pourrait-il le faire ? La réponse est ici encore résolument négative dans la mesure où le droit du licenciement est d'ordre public strict. Un texte conventionnel pourrait évidemment améliorer la situation du salarié mais en aucune manière réduire les droits que celui-ci tire de la loi.

Resterait alors l'hypothèse d'une modification législative étant précisé que cette modification porterait, non sur l'indemnisation du licenciement, mais sur l'indemnisation du préjudice résultant de l'absence de motif réel et sérieux ou, pour reprendre une terminologie plus ancienne, d'un abus du droit de rompre le contrat.

5. C. trav., art. L 122-14-7.

6. RJS $2005, \mathrm{n}^{\circ} 594$. 
Le droit à réparation du préjudice subi est un principe du droit de la responsabilité. Dès lors, sauf à interdire au salarié le droit de faire juger que le licenciement n'est pas fondé sur un motif réel et sérieux, ce qui n'est guère compatible avec le droit au juge internationalement protégé, on ne voit guère comment un salarié pourrait accepter par avance de ne pas saisir le juge pour obtenir une indemnisation consécutive à un licenciement fondé sur un motif illégitime.

Or, l'existence d'un plafond d'indemnisation en cas de licenciement non fondé sur une cause réelle et sérieuse conduirait nécessairement à empêcher le salarié d'obtenir la réparation intégrale de son préjudice. Cela reviendrait en quelque sorte à mettre en place législativement une sorte de franchise comme en matière de contrat d'assurance.

Faire prendre en charge cette indemnisation par un organisme public et mutualiser le coût des licenciements illégitimes ne change en rien le fait que le salarié garderait le droit de saisir le juge afin d'obtenir une indemnisation complémentaire liée au comportement de l'employeur. L'exemple de l'évolution de la jurisprudence en matière de réparation des accidents du travail est de ce point de vue très évocateur. L'instauration d'une indemnisation automatique et forfaitaire des pathologies professionnelles a conduit au développement des contentieux sur la reconnaissance de la faute inexcusable qui permet précisément de sortir des mécanismes de forfaitisation. Si le but est de déjudiciariser le licenciement, le résultat pourrait bien être totalement inverse de l'objectif recherché.

\section{Les dispositions introduites en seconde lecture à la Loi Macron}

Des dispositions ajoutées à la Loi Macron lors de sa seconde lecture à l'Assemblée nationale en juin 2015 visaient à encadrer le montant des indemnités prononcées pour les licenciements dénués de cause réelle et sérieuse, par des barèmes diversifiés selon la taille de l'entreprise concernée. La mesure envisagée présentait des risques juridiques. Tout d'abord, comme développé plus haut, la fixation forfaitaire (ou maximum) des indemnités allouées par le jugement peut poser un problème au regard du principe de réparation intégrale du préjudice. Il serait plus prudent, comme nous le proposons, de distinguer l'absence de cause sérieuse pour laquelle le forfait barémisé peut se concevoir et le licenciement abusif où resterait en vigueur la réparation intégrale du préjudice. Certaines diversités envisagées des barèmes présentaient un autre risque juridique. La différence de plafond d'indemnisation liée à l'ancienneté peut se justifier, d'autant que c'est déjà le cas pour les indemnités, tant légales que conventionnelles, dues lorsque la rupture repose sur une cause sérieuse. Par contre, différencier, comme il était envisagé, les indemnités en fonction de la taille de l'entreprise était périlleux dès lors qu'il s'agit de dommages et intérêts. On cherchera en vain l'argument pour justifier que le préjudice subi par le salarié est moindre s'il travaille dans une TPE que dans une entreprise dont 
l'effectif est plus important. Cette dernière disposition a d'ailleurs été censurée par le Conseil constitutionnel.. Nous appelons à la fois à plus d'ambition et moins de prise de risque dans l'adaptation du Code du travail, au besoin d'une meilleure conciliation entre protection des travailleurs et efficacité économique.

Cet encadré reprend des éléments de l'Annexe 1 de Barthelemy, Cette et Verkindt (2006).

\section{Internaliser en partie le coût social des licenciements}

Les propositions du rapport Cahuc et Kramarz (2005) sont à cet égard les suivantes :

- Paiement d'une indemnité de licenciement croissante avec l'ancienneté, mais décroissante en pourcentage des salaires versés par l'entreprise depuis l'embauche du salarié. Ainsi calibrée, cette indemnité inciterait l'entreprise à stabiliser l'emploi et à garder des salariés plutôt qu'à en changer. Par exemple, toutes choses égales par ailleurs, l'indemnité serait au total nettement plus forte dans le cas de 2 salariés se succédant et licenciés chacun au bout de 2 ans que dans le cas d'un salarié licencié au bout de 4 ans. La proposition visant à accroître les indemnités de rupture avec l'ancienneté est surprenante... car c'est déjà le cas ! En fait apparaît une confusion entre les indemnités (légales et conventionnelles) contrepartie de l'utilisation régulière du droit de licencier et les dommages et intérêts dus en plus et les conséquences du recours abusif ou irrégulier à ce droit ;

- Paiement d'une contribution de solidarité, proportionnelle au salaire versé par l'entreprise depuis l'embauche du salarié. Cette contribution est légitimée par la disproportion actuelle entre le coût de l'indemnisation chômage des personnes ayant acquis leur droit dans des emplois précaires et les cotisations chômage prélevées sur l'emploi précaire. Elle vise à internaliser le coût social du licenciement, en contribuant : (i) A financer la prise en charge du reclassement du salarié, si l'activité de reclassement est assurée par des tiers professionnels et non par l'entreprise qui licencie. Si l'entreprise prend la charge de ce reclassement, bien entendu cette part 
de la contribution de solidarité ne serait pas due; (ii) A financer le coût des prestations chômage.

Le rapport de Blanchard et Tirole (2003) ${ }^{7}$ préconise aussi d'associer taxes de licenciement, visant à responsabiliser les entreprises aux coûts sociaux de leurs licenciements, et indemnités de licenciement dédommageant des coûts individuels et par exemple psychologiques. Camdessus $(2004)^{8}$ propose une responsabilisation de l'entreprise via l'instauration d'un système de bonus-malus sur les cotisations sociales selon l'historique de chaque entreprise dans sa gestion de l'emploi, un peu à la manière de ce qui existe aux États-Unis de façon variée selon les États.

En fait, on commet ici un autre contresens, celui d'associer toute rupture de contrat de travail à une cause économique. Or, le nombre de licenciements pour cause personnelle est bien plus élevé que celui lié à la nécessaire adaptation du volume et de la nature des emplois à l'évolution du contexte économique. Une nouvelle fois doit être posée la question : veut-on que l'employeur puisse licencier sans motif légitime au mépris notamment de la charte des droits fondamentaux de l'Union européenne?

L'ANI du 11 janvier 2013 s'est invité dans ce débat en proposant de majorer les taux de cotisations chômage employeurs sur les CDD de moins de trois mois, cette majoration étant même rendue plus importante sur les CDD de moins d'un mois. L'ANI a également proposé d'annuler durant trois mois le taux de cotisations chômage employeurs sur les jeunes de moins de 25 ans embauchés en CDI. Ces deux dispositions, reprises dans la loi du 14 juin 2013, ont pour

7. Par exemple : «Les taxes de licenciement représentent pour nous l'instrument essentiel de responsabilisation financière des entreprises. Nous ne sommes pas cependant en faveur de l'élimination des indemnités de licenciement qui peuvent, au moins partiellement, compenser les coûts psychologiques de la perte de l'emploi (par opposition au coût du chômage lui-même). Comme ces coûts psychologiques sont en général faibles pour les salariés avec peu d'ancienneté, ceci suggère un barème d'indemnités augmentant avec l'ancienneté. Dans cette optique, le barème légal actuel paraît raisonnable » (Blanchard et Tirole, 2003, p. 46).

8. «Un système de bonus-malus sur les cotisations sociales payées par l'entreprise tenant compte du nombre d'embauches et de licenciements au cours de l'année pourrait être instauré. L'idée est d'inciter à l'embauche, et de "désinciter» les licenciements, en adoptant une approche économique, i.e. plus rapidement et plus sûrement que par la voie judiciaire. En effet, à l'heure actuelle, les contributions des entreprises à la caisse d'assurance chômage sont basées sur la masse salariale, et sont donc indépendantes du comportement des entreprises en matière de licenciement. L'entreprise ne tient donc pas compte lorsqu'elle licencie du coût de ce licenciement pour la collectivité. Pour inciter l'entreprise à la prendre en compte, un dispositif financier convenablement calibré a tout son sens » (Camdessus, 2004, p. 98). 
finalité explicite de réduire la dualité sur le marché du travail. Elles permettront de sécuriser davantage les parcours professionnels en désincitant financièrement les licenciements hâtifs.

Si l'on peut se féliciter d'avancées récentes qui vont contribuer à dissuader le recours abusif aux CDD, il semble préférable, comme indiqué dans la partie 2, de mobiliser les acteurs de l'activité économique sur cet aspect plutôt que de supprimer les CDD, ce à quoi revient concrètement le contrat unique. A cette fin, notre proposition (Cf. Barthelemy et Cette, 2013) est : (i) de taxer davantage, par une majoration de la contribution de solidarité évoquée ci-dessus pour les CDI, le recours à l'emploi précaire ; (ii) d'abaisser cette majoration quand le recours à l'emploi précaire est encadré par accord collectif. Cette disposition responsabiliserait les partenaires sociaux sur la question de l'emploi précaire en les amenant à discuter des besoins de l'entreprise et des attentes des salariés et, ce faisant, dynamiserait la négociation collective et favoriserait le développement du droit conventionnel.

Une autre suggestion pertinente est celle avancée par Coquet et Sylvain (2007) et Coquet (2011). Elle consiste à préconiser que le taux de cotisations chômage employeurs soit pour chaque salarié dégressif avec son ancienneté dans l'entreprise. Ces propositions nous paraissent pertinentes. Elles permettent de sécuriser davantage les parcours professionnels en « désincitant » financièrement les licenciements rapides et le recours trop fréquent à des emplois facteurs de précarité. Il va de soi que des dispositions conventionnelles pourraient majorer les primes de licenciement prévues par la réglementation. Dans la logique de nos propositions, deux précisions supplémentaires sont à apporter concernant les CDD :

- La prime de précarité versée en fin de contrat se substitue à l'indemnité de licenciement. Son calibrage devrait être financièrement plus avantageux pour les salariés, pour les raisons qui viennent d'être évoquées ;

- Concernant la contribution de solidarité, il serait préjudiciable à l'efficacité économique de limiter par un tel surcoût le recours aux emplois précaires de façon uniforme pour toutes les activités économiques. Il n'est pas souhaitable que la loi fixe les bons et mauvais recours à l'emploi précaire. Dans notre approche, il faudrait que cette majoration soit atténuée dans le cas où le recours à l'emploi précaire serait 
encadré par un accord collectif majoritaire. Toutefois, il ne faut pas perdre de vue dans cette analyse que les indemnités de mise à la retraite sont également fonction de l'ancienneté et que leur montant légal est identique à celui de l'indemnité légale de licenciement.

\section{Quelques remarques conclusives}

Le reclassement des salariés licenciés est une préoccupation majeure, qui doit être financée par la solidarité quelle que soit la dimension de l'entreprise. Les efforts de reclassements et les plans de sauvegarde de l'emploi ne doivent plus être une obligation pour les entreprises, souvent non préparées et qualifiées pour cela, et dont les services du personnel sont souvent déjà mobilisés pour préparer et mettre en œuvre les réorganisations internes associées aux licenciements. Pour autant, la contribution de solidarité devrait pouvoir être réduite par décision des services de l'emploi dans les cas d'entreprises développant un réel effort de reclassement. Dans les autres cas, elle financerait ces efforts de reclassement qui seraient assurés par des professionnels. Rappelons ici que l'employeur est tenu (du fait de les articles L6321-1 et -2 du Code du travail) de tout mettre en œuvre pour maintenir l'employabilité de chaque salarié, cette obligation étant similaire à celle lui incombant en matière de santé et de sécurité.

Les trois rapports évoqués préconisent l'instauration d'un contrat unique se substituant à la juxtaposition actuelle des CDD et des CDI. Le principal risque est que cette réforme soit perçue comme LA réforme du droit du travail français. Et ce serait catastrophique car cela gèlerait toute initiative de refondation du droit social.

La question qui se pose semble plutôt être: concernant le marché du travail, quelles sont les priorités? Les dénonciations d'un droit réglementaire trop complexe, contraignant et homogène sont fréquentes. Ce droit a sans doute assez peu bridé la croissance d'une économie en rattrapage dans les décennies 1950, 1960 et 1970 . Il a pu être adapté à l'absorption de certains chocs importants, comme par exemple des chocs de population active. Mais, outre que sa rigidité et sa complexité ont été augmentées depuis, il paraît davantage inhibant aujourd'hui, compte tenu des 
conditions de production actuelles et des chocs que nous connaissons de nos jours. En particulier, il est sans doute peu adapté à celui constitué par l'émergence des technologies de l'information et de la communication, dont la mobilisation pleine et entière des performances productives appelle des formes de flexibilité particulières. La complexité du droit réglementaire est par ailleurs souvent évoquée comme source d'inégalités puisque, comme indiqué par Cahuc et Kramarz (2005) ${ }^{9}$, elle pénalise les moins bien lotis. Elle est aussi facteur d'ineffectivité de la loi que l'on ne comprend plus.

La seule réforme ambitieuse est celle qui vise à abandonner un droit du travail prioritairement d'essence réglementaire, celui dans lequel le projet de contrat unique reste enfermé. La priorité est la contraction du droit réglementaire et, simultanément, le développement du droit conventionnel, pour favoriser une flexibilité négociée, gérée par les partenaires sociaux. Ceci d'autant que la suppression du CDD ne réglerait pas, comme il est parfois dit, la question de la précarité qui serait alors appréhendée autrement. Le développement dérogatoire du droit conventionnel est la seule voie qui permet de renforcer simultanément l'efficacité économique et la fonction protectrice du droit social ${ }^{10}$.

On peut se hasarder à taxer de naïve l'idée qu'en supprimant le CDD on écarte les conséquences négatives sur le terrain de la précarité. C'est par solution de facilité et parce qu'ils font le même contresens que les promoteurs de l'idée de contrat unique que les banquiers sont réticents à prêter aux titulaires de CDD. Étant donné que ce n'est pas en soi la nature du contrat qui génère de la précarité, ils rechercheront d'autres critères pour attester d'un tel état ! Au demeurant, un banquier hésite-t-il à prêter à une star du

9. Cahuc et Kramarz (2005, p. 135) indiquent ainsi : « Nous venons de souligner que la réglementation française de la protection de l'emploi est, à plusieurs égards, particulière. Malheureusement, cette particularité est considérée par beaucoup comme une faiblesse de notre appareil réglementaire qui est excessivement complexe et difficile à appliquer. Cette complexité aboutit à un contournement systématique du droit, à l'origine de profondes inégalités de traitement qui défavorisent les moins bien lotis. Cette réglementation conduit à limiter les possibilités d'anticipation des restructurations ainsi qu'à limiter la participation des représentants de salariés ». Nous partageons pleinement ces constats, quoi qu'ils nous amènent logiquement à écarter la proposition de contrat unique, qui prolonge une approche réglementaire, pour préconiser plutôt une gestion de la réduction de la complexité par les partenaires sociaux euxmêmes, comme indiqué plus loin.

10. Cette analyse est développée dans Barthelemy et Cette (2015). 
sport professionnel dont le contrat est impérativement à durée déterminée, souvent pour une seule saison?

Enfin, dans toutes les disciplines juridiques et dans le monde entier coexistent deux formes de contractation: l'une à temps défini et l'autre pour une durée non limitée assortie de règles de dénonciation. Pourquoi le contrat de travail serait-il le seul type de contrat à ne pas être en conformité avec cette exigence qui puise au demeurant sa force dans des principes fondamentaux du droit? On ne peut pas procéder à des constructions qui, consciemment ou pas, reposent sur l'idée que l'économie est une science et que le droit est un instrument au service des gouvernements pour la mettre en ouvre, ceci d'autant que, ce faisant, on limite le champ du droit à la loi qui n'en est qu'une source. Le contrat est d'autant plus source de droit que la liberté contractuelle est inscrite dans l'article 4 de la Déclaration des droits de l'Homme et du citoyen annexée à la Constitution française mais aussi dans la Conv. EDH !

\section{Références bibliographiques}

Barthélémy J., G. Cette et P.-Y. Verkindt, 2006, Contrat de travail, sécurisation des parcours professionnels et efficacité économique, Mimeo, Rapport au Conseil d'orientation de l'emploi, 26 décembre.

Barthélémy J., G. Cette et P.-Y. Verkindt, 2007a, «À propos du contrat unique ", La Semaine juridique, 6 février, $6: 3-4$.

Barthélémy J., G. Cette et P.-Y. Verkindt, 2007b, «À propos du contrat unique » in Le contrat de travail, Centre d'Études de l'Emploi (éd.), La Découverte, Collection Repères, 107-118.

Barthélémy J. et G. Cette, 2015, Réformer le droit du travail, Rapport pour la Fondation Terra Nova, Éditions Odile Jacob.

Blanchard O. et J. Tirole, 2003, «Protection de l'emploi et procédures de licenciement », Rapport du Conseil d'analyse économique, 44.

Cahuc P. et F. Kramarz, 2005, De la précarité à la mobilité : Vers une Sécurité sociale professionnelle, Rapport au ministre des Finances et de l'industrie et au ministre de l'Emploi, du travail et de la cohésion sociale, La Documentation française.

Camdessus M., 2004, Le sursaut - Vers une nouvelle croissance pour la France, La Documentation française.

Coquet B. et A. Sylvain, 2007, «L'indemnisation du chômage : éléments pour une réforme », Sociétal, 55. 
Coquet B., 2011, L'assurance-chômage et le marché du travail contemporain, Thèse de Doctorat, Université d'Aix-Marseille.

Karaquillo J.-P., 2015, Statuts des sportifs, Rapport remis à Thierry Braillard, Secrétaire d'État aux Sports, le 18 février. 
